\title{
Concurrence of sarcoidosis and lung cancer: a diagnostic dilemma
}

$\mathrm{T}$ he development of lung cancer in a patient with pulmonary sarcoidosis is recognised, but differentiation between the two conditions is not always easy. ${ }^{1}$ The concurrence of pulmonary sarcoidosis and primary or secondary lung tumours can cause a diagnostic dilemma and make preoperative staging difficult. ${ }^{2}$ We describe the use of positron emission tomography (PET) to confirm the diagnosis and provide accurate staging in a patient with sarcoidosis and lung cancer.

A 68 year old man with a known pulmonary sarcoidosis presented with haemoptysis. Serial chest radiographs over a number of years had shown apical opacities in both lungs and mediastinal widening due to sarcoidosis. His current chest radiograph showed further enlargement of the apical opacity in the right lung. A computed tomographic (CT) scan was performed (fig 1). Fibreoptic bronchoscopy and biopsy samples showed non-small cell carcinoma in the right upper lobe.

We could not accurately stage his nodal or metastatic status from the CT scan alone. Mediastinoscopy and lymph node biopsy showed sarcoidosis, but there was the possibility of a sampling error so a benign biopsy could not exclude tumour in nodes that were not sampled. Furthermore, the lesion in the left upper lobe was not visible endobronchially, and attempts at

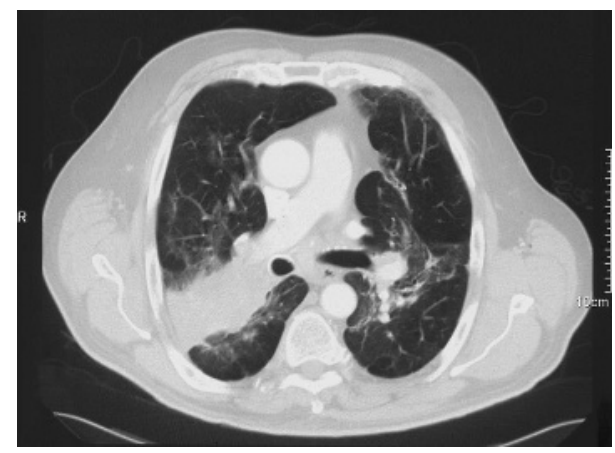

Figure 1 Chest CT scan showing extensive mediastinal lymphadenopathy (up to $3 \mathrm{~cm}$ ), a $5.5 \mathrm{~cm}$ mass in the right upper lobe, and a smaller $2.3 \mathrm{~cm}$ mass in the left upper lobe.

\section{Learning points}

- CT scanning is a good predictor of mediastinal staging in lung cancer but, in the presence of sarcoidosis, it cannot alone identify the nature of lymphadenopathy or a contralateral radiographic abnormality.

- PET scanning can differentiate between benign and malignant pulmonary lesions due to semi-quantitative assessment by differential standardized uptake value (SUV) of FDG. It is able to determine the nodal status to help preoperative staging. The sensitivity and specificity of PET is $96 \%$ and $77 \%$ respectively for diagnosing malignant nodules. It is also more accurate than CT scanning for staging mediastinal nodal involvement (sensitivity $89 \%$, specificity $94 \%$ ).

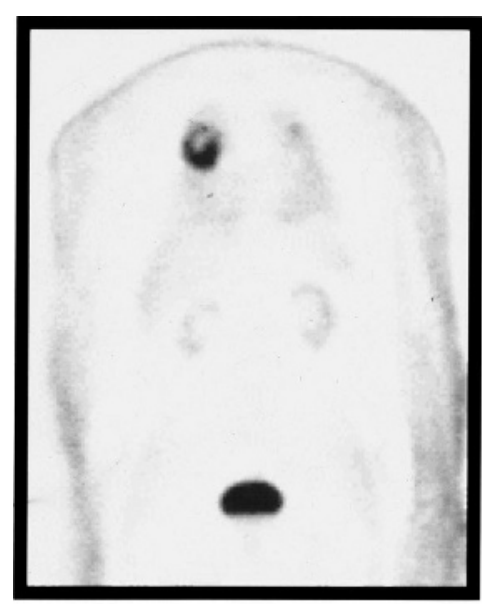

Coronal

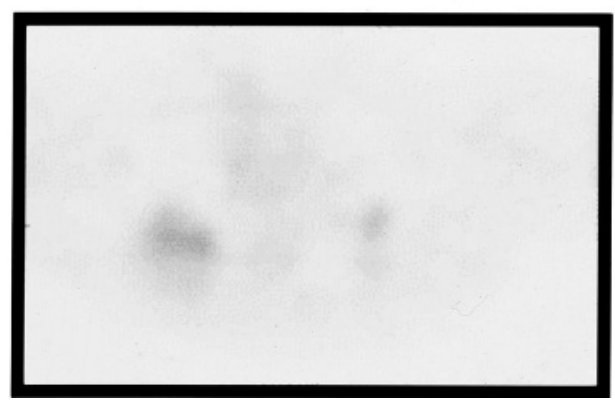

Transaxial

Figure 2 PET scan showing significantly enhanced uptake in the right upper lobe (SUV 9.5) with less uptake in the left lobe (SUV 2.0).

transbronchial needle aspiration cytology and CT guided percutaneous transthoracic needle aspiration were non-diagnostic. We therefore performed a PET scan (fig 2) which was interpreted as malignancy in the right upper lobe based on a high standardised uptake value (SUV) of FDG, ${ }^{3}{ }^{4}$ with the other areas being entirely in keeping with sarcoidosis.

The patient underwent thoracotomy and right upper lobectomy. Histological examination showed a completely resected moderately differentiated squamous cell carcinoma without local or regional lymph node involvement (pT2NOM0).

S Kumar, S Baghdadi, A R J Cale Department of Cardiothoracic Surgery, East Yorkshire Cardiothoracic Center, Castle Hill Hospital, Cottingham, Hull, UK
Contic Correspondence to: Dr S Kumar, Department of Cardiothoracic Surgery, Leeds General Infirmary, Leeds LS1 3EX, UK; sanjaykr33@yahoo.co.uk,

\section{REFERENCES}

1 Seerholm N, Vesbo J, Viskum K. Risk of malignant neoplasms in patients with pulmonary sarcoidosis. Thorax 1997;53:892-4.

2 Detterbeck FC, DeCamp MM Jr, Kohman U, et al. Lung cancer. Invasive staging: the guidelines. Chest 2003;123(1 Suppl): 167-75S.

3 Gould MK, Maclean CC, Kuschner WG, et al. Accuracy of positron emission tomography for diagnosis of pulmonary nodules and mass lesions: a metaanalysis. JAMA 2001;21;285:914-24.

4 Yang SN, Liang JA, Lin FJ, et al. Differentiating benign and malignant pulmonary lesions with FDG-PET. Anticancer Res 2001;21:4153-7. 